\title{
Charge identification in PAMELA: preliminary results on light nuclei.
}

\author{
Rita CARBONE* \\ INFN Napoli and University of Rome Tor Vergata \\ E-mail: carboneena.infn.it
}

on behalf of PAMELA collaboration

\begin{abstract}
The study of the light-nuclei component of the cosmic radiation is strictly connected to a better understanding of the propagation properties, which has great importance for the study of signatures of new physics in CRs. For example, indirect signals of dark matter pairs annihilating in the halo of our Galaxy could be found in antiproton, antideuteron or positron CRs but this research is limited by the uncertainties in the propagation parameters and fluxes of charged particles located in the whole diffusive halo. The PAMELA experiment will contribute to this issue measuring light nuclei fluxes and ratios with good accuracy, also in some unexplored energy range. Some preliminary results on discrimination capability of the subdetectors will be presented in this work.
\end{abstract}

Identification of dark matter 2008

August 18-22, 2008

Stockholm, Sweden

${ }^{*}$ Speaker. 


\section{The PAMELA experiment.}

PAMELA is a satellite-borne experiment [1] built to detect charged particles in cosmic rays with particular attention to antiparticles. PAMELA is in orbit from June 15th 2006. All the detectors are working nominally and analysis is in progress to achieve many scientific goals: principally the search for antimatter in primary radiation, the search for dark matter sources but also the measurement of fluxes and ratios of the different components of the cosmic radiation and the study of interactions of the radiation itself with Sun and Magnetosphere.

To reach all these scopes, PAMELA is composed by several instruments perfectly integrated: the experimental setup allows us to operate precise momentum measurements, $d E / d x$ and charge spectrum reconstruction as well as investigation of many other topics. Details about the science of PAMELA, as well as the detector, its general status and some preliminary results, will be described in another paper in this conference [2].

\section{Study of the Nuclei component of cosmic rays with PAMELA.}

PAMELA will provide new high precision measurements about primary and secondary fluxes and abundances of light nuclei (at least up to Carbon) in cosmic radiation, the way to put constrains on the current diffusion models of cosmic rays in the Galaxy. In fact, the ratios of spallogenic nuclei (B, Be and Li)to mostly primary nuclei such as Carbon are sensitive to the amount of material traversed by GCRs from the source to detection at Earth[3].

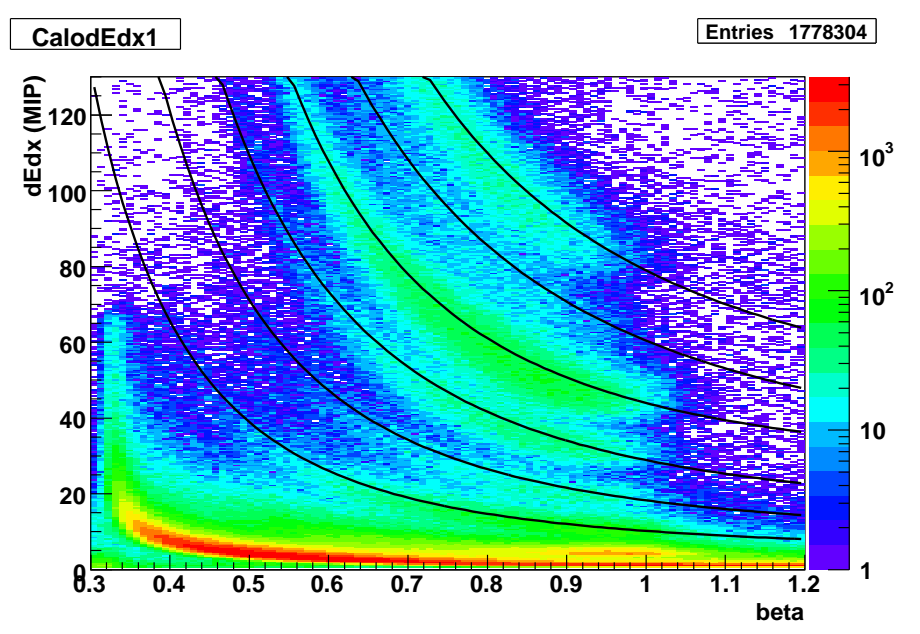

Figure 1: Plot of the energy released inside the first plane of the calorimeter as a function of $\beta$ (measured by the ToF system). According to Bethe-Block equation, different bands for each family of nuclei are visible. Bands from $\mathrm{Li}$ to $\mathrm{O}$ are fitted by Bethe-Block functions.

The identification of nuclei events is operated by PAMELA by means of measurements of the energy loss inside the magnetic spectrometer, the Time-of-Flight system and the calorimeter. In fact, plotting the measurements of the energy released inside the sensitive areas of the detectors as a function of the velocity of the particles (which can be derived by $\beta$ measurements from ToF or rigidity measurements from spectrometer), we see that points related to nuclei of different $\mathrm{Z}$ fall 


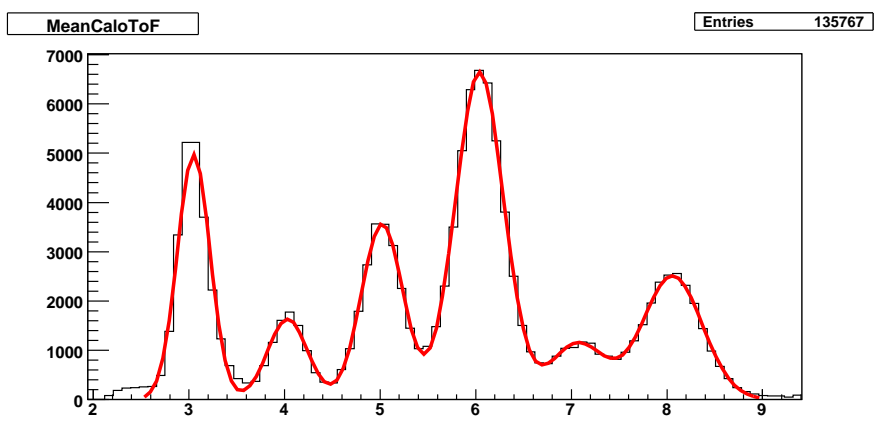

Figure 2: $\mathrm{Z}$ distribution as obtained by combining measurements from $\mathrm{ToF}$ and calorimeter for events which do not produce fragments inside the detector (in all energy range).

into different bands according to Bethe-Block formula (see also figure 1); by fitting these bands it is possible to assign to every $\mathrm{Z}$ a mean value of charge deposit at the minimum of ionization. This way we can reconstruct a $\mathrm{Z}$ spectrum from each sub-detector or from the combination of two or more of them.

\section{Resolution on Nuclei identification.}

To estimate the resolution on Nuclei identification and discrimination of PAMELA, we choose to combine measurements from both $\mathrm{ToF}$ and calorimeter; this way we are able to select a sample of events that do not fragment inside the instrument (by requiring that the values of $Z$ measured by the two sub-detectors for the same event have to be compatible) with a sufficiently high statistic that does not suffer problems of saturation in a significant amount (at least up to Carbon). The $\mathrm{Z}$ spectrum obtained by using those requirements is plotted in figure 2 ; in table 1 there are the estimated resolutions for each nuclei family. Notice that to obtain this "mean" resolution we take into account nuclei events in all energy range, but it is obvious that such resolution improves with increasing energy, as it is possible to see in figure 3 .

\begin{tabular}{|c|c|c|}
\hline \hline Nuclei & $Z$ & $\sigma_{Z}$ \\
\hline $\mathrm{Li}$ & 3 & 0.17 \\
\hline $\mathrm{Be}$ & 4 & 0.21 \\
\hline $\mathrm{B}$ & 5 & 0.22 \\
\hline $\mathrm{C}$ & 6 & 0.25 \\
\hline $\mathrm{N}$ & 7 & 0.3 \\
\hline $\mathrm{O}$ & 8 & 0.3 \\
\hline \hline
\end{tabular}

Table 1: Resolution on charge identification for Nuclei up to Oxygen as obtained by combining measurements from $\mathrm{ToF}$ and calorimeter. 

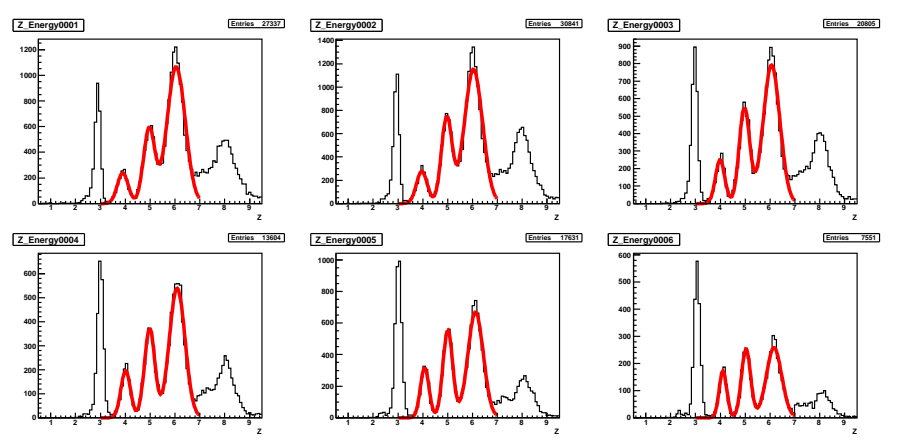

Figure 3: $\mathrm{Z}$ distribution in different energy bins. In the first plot energy varies from $200 \mathrm{MeV}$ to $400 \mathrm{MeV}$; in the second from $400 \mathrm{MeV}$ to $600 \mathrm{MeV}$; in the third from $600 \mathrm{MeV}$ to $800 \mathrm{MeV}$; in the 4th from $800 \mathrm{MeV}$ to $1 \mathrm{GeV}$; in the 5th from $1 \mathrm{GeV}$ to $1.5 \mathrm{GeV}$; in the last from $1.5 \mathrm{GeV}$ to $2.5 \mathrm{GeV}$. Gaussian fits for Be, B and $\mathrm{C}$ distributions are superimposed to the graph. In this case energy is reconstructed by ToF system alone; it is possible to investigate the higher energy range using measurements from magnetic spectrometer. To estimate abundances and ratios, fragmentation inside the detector and efficiency of the sub-detectors must be taken into account.

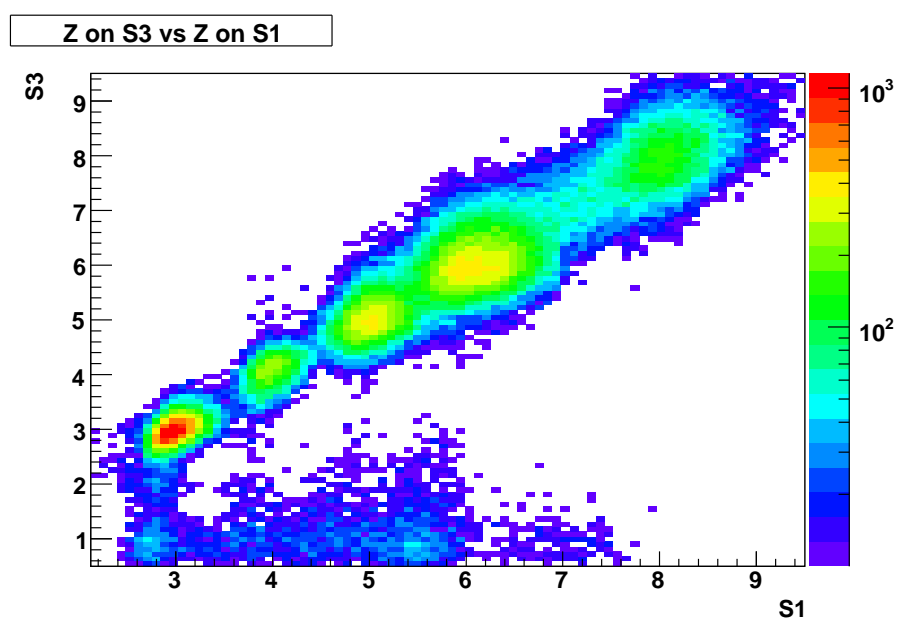

Figure 4: Scatterplot of $\mathrm{Z}$ as reconstructed by the lower plane of the ToF system as a function of $\mathrm{Z}$ as reconstructed by the upper plane: fragmentation events are visible in the lower part of the plot.

Notice, also, that the spectrum in figure 2 is not valid to estimate abundances because it has to be corrected for fragmentation inside the detector (see also next section) and for the efficiency of the sub-detectors.

\section{Estimation of fragmentation rate inside the detector.}

As it is evident looking at the scatterplot in figure 4, many fragmentation events occur inside the detector. A quantitative evaluation of such fragmentation rate for each nuclei family is necessary to tune Monte Carlo, the way to avoid systematic errors in the estimation of efficiency of the sub-detectors. 


\section{References}

[1] P. Picozza, et al., Astroparticle Physics 27 296-315 (2007)

[2] M. Boezio on behalf of PAMELA collaboration, Proceeding of this Conference

[3] A. Castellina, F. Donato, Astroparticle Physics 24 146-159 (2005) 